Published in final edited form as:

Microb Pathog. 2016 March ; 92: 60-67. doi:10.1016/j.micpath.2015.12.006.

\title{
The modA10 phasevarion of Nontypeable $H$. influenzae R2866 regulates multiple virulence-associated traits
}

\author{
Timothy M. VanWagoner ${ }^{1}$, John M. Atack ${ }^{4}$, Kevin L. Nelson ${ }^{2}$, Hannah K. Smith ${ }^{2}$, Kate L. \\ Fox $^{3}$, Michael P. Jennings ${ }^{4}$, L. Stull Terrence ${ }^{1}$, and Arnold L. Smith ${ }^{2,}$ \\ ${ }^{1}$ Department of Pediatrics, University of Oklahoma Health Sciences Center, Oklahoma City, \\ 73104, OK, USA \\ ${ }^{2}$ Center for Global Infectious Disease Research, Seattle Children's Hospital, Seattle, WA, 98105, \\ USA \\ ${ }^{3}$ School of Chemical and Molecular Bioscience, University of Queensland, St. Lucia, Brisbane, \\ Queensland 4072, Australia \\ 4 Institute for Glycomics, Griffith University, Gold Coast, Queensland 4222, Australia
}

\begin{abstract}
Non-typeable Haemophilus influenzae (NTHi) is a human restricted commensal and pathogen that elicits inflammation by adhering to and invading airway epithelia cells: transcytosis across these cells can result in systemic infection. NTHi strain R2866 was isolated from the blood of a normal 30-month old infant with meningitis, and is unusual for NTHi in that it is able to cause systemic infection. Strain R2866 is able to replicate in normal human serum due to expression of lgtC which mimics human blood group $p^{k}$. R2866 contains a phase-variable DNA methyltransferase, modA10 which switches ON and OFF randomly and reversibly due to polymerase slippage over a long tetrameric repeat tract located in its open reading frame. Random gain or loss of repeats during replication can results in expressed (ON), or not expressed (OFF) states, the latter due to a frameshift or transcriptional termination at a premature stop codon. We sought to determine if the unusual virulence of R2866 was modified by modA10 phase-variation. A modA10 knockout mutant was found to have increased adherence to, and invasion of, human ear and airway monolayers in culture, and increased invasion and transcytosis of polarized human bronchial epithelial cells. Intriguingly, the rate of bacteremia was lower in the infant rat model of infection than a wild-type R2866 strain, but the fatality rate was greater. Transcriptional analysis comparing the modA10 knockout to the R2866 wild-type parent strain showed increased expression of genes in the modA10 knockout whose products mediate cellular adherence. We conclude that loss of ModA10 function in strain R2866 enhances colonization and invasion by increasing expression of genes that allow for increased adherence, which can contribute to the increased virulence of this strain.
\end{abstract}

\footnotetext{
* Corresponding author, arnold.smith@ seattlechildrens.org.

Publisher's Disclaimer: This is a PDF file of an unedited manuscript that has been accepted for publication. As a service to our customers we are providing this early version of the manuscript. The manuscript will undergo copyediting, typesetting, and review of the resulting proof before it is published in its final citable form. Please note that during the production process errors may be discovered which could affect the content, and all legal disclaimers that apply to the journal pertain.
} 


\section{Keywords}

Haemophilus influenzae; Pathogenesis; Phase variation; DNA methylase; Virulence

\section{Introduction}

Non-typeable Haemophilus influenzae (NTHi) is a human-restricted Gram-negative bacterium that resides in the human oropharynx as a commensal, but is also associated with infections of the nasopharynx and respiratory tract such as pneumonia, otitis media, chronic bronchitis and sinusitis; occasionally causing invasive disease. It does not express exotoxin but, causes disease by adhering to and invading cells of the respiratory mucosa, which elicits an inflammatory response [1]. Understanding the mechanisms of adherence and invasion will permit preventative strategies to be developed.

Many bacterial pathogens contain phase-variable genes. Phase variation is the random and reversible switching of gene expression, and is typically associated with bacterial surface factors such as LOS [1,2], iron acquisition genes [3,4], and adhesins [5]. The random ON/OFF switching of these genes results in phenotypically diverse populations that can rapidly adapt to host environments and evade immune responses [6]. Phase variation is achieved by many mechanisms, but in certain genera it results from slipped-strand mispairing over simple sequence repeats (SSRs) that follow the open-reading frame of the gene in question. Several human-restricted bacterial pathogens, including NTHi, contain methyltransferases, encoded by mod genes, associated with type III restriction-modification systems which contain SSRs. A deletion or insertion in the SSR tract in the mod gene can cause a frameshift in $\bmod$ resulting in either translation of a functional truncated $\bmod (\mathrm{ON})$, or a premature transcriptional termination $(\mathrm{OFF})$.

We have previously reported that phase variation of mod in several clinically important bacterial pathogens results in global changes in gene expression due to phase variable methylation of certain sites in the genome. These phase variable regulons, or phasevarions [7], control expression of surface antigens and virulence factors, leading to altered phenotypes between Mod ON and OFF variants. Phasevarions have been studied in $H$. influenzae (ModA; [8,9], N. gonorrhoeae and N. meningitidis (ModA, ModB and ModD; (40-42); H. pylori (ModH; [13] and M. catarrhalis (ModM [14]). The structure of each class of Mod proteins (ModA, B, D, H, and M) are highly conserved, apart from the central DNA recognition domain (DRD) that dictates methylation specificity [15]. Different mod alleles have different DRD encoding sequences, yielding Mod proteins containing different DRDs methylating different DNA sequences [7].

NTHi contains twenty-one modA alleles, all containing distinct DRDs, and all methylating distinct DNA sequences and therefore controlling distinct phasevarions. However, over $60 \%$ of all NTHi clinical isolates contain just five phase variable $\operatorname{modA}$ alleles - $\operatorname{modA2}$, 4, 5, 9 and 10 [8], with $\operatorname{modA10}$ is present in approximately $15 \%$ of all clinical isolates, making it second only in prevalence in clinical NTHi samples to modA2 [8]. The modA2 ON state was shown to be preferentially selected for in an in vivo chinchilla model, with all five of the most prevalent $\bmod A$ alleles regulating expression of multiple proteins including a number 
of current and putative vaccine candidates [8]. Strain R2866 is unusual amongst NTHi in that it was isolated from the blood of a normal child with meningitis [16]. We have shown that strain R2866 cultures with $90 \% \operatorname{modA} 10$ ON cells have increased expression of OMP P6 and OMP P6 in comparison to cultures with modA10 predominantly OFF [8].

In order to further characterize the modA10 phasevarion, identify genes involved in virulence and host adaptation regulated by $\bmod A 10$, and relate these properties to the increased invasive capacity of strain R2866 containing this $\bmod A$ allele, we performed phenotypic and transcriptomic studies of strain R2866 containing the wild-type and mutated $\bmod 10$ allele.

\section{Materials Methods}

\subsection{Bacteria and growth conditions}

Table 1 details the $H$. influenzae strains used in this study. All $H$. influenzae strains were stored at $-70^{\circ} \mathrm{C}$ in $10 \%$ skim milk and sub-cultured o nto chocolate agar (Difco) containing $1 \%$ haemoglobin (BD Biosciences) 5,000 U of bacitracin and 1\% GCHI Rehydrating Solution (Remel, Lenexa, KS) containing Ribostamycin $25 \mu \mathrm{g} / \mathrm{ml}$; Kanamycin $30 \mu \mathrm{g} / \mathrm{ml}$; or Streptomycin $250 \mu \mathrm{g} / \mathrm{ml}$ as necessary, and incubated overnight at $37^{\circ} \mathrm{C}$ in air. Since most $H$. influenzae will develop high-level, chromosomal streptomycin resistance in a single step, we regularly used streptomycin resistant derivatives, particularly when construction was occurring on the floor housing the laboratory..

Chemically defined medium (CDM) for the growth of $H$. influenzae was prepared as follows: $191 \mathrm{ml}$ of RPMI 1640 with L-glutamine and $25 \mathrm{mM}$ HEPES, pH 7.26 (InVitrogen), $2 \mathrm{ml}$ of a $100 \mathrm{mM}$ MEM sodium pyruvate solution (InVitrogen), $2 \mathrm{ml}$ of $\beta$-NAD ${ }^{+}$stock, 4 $\mathrm{ml}$ of heme-L-histidine stock, $10 \mathrm{ml}$ of a $2-\mathrm{mg} / \mathrm{ml}$ uracil solution (Sigma) dissolved in $0.1 \mathrm{~N}$ $\mathrm{NaOH}$, and $20 \mathrm{ml}$ of a $20-\mathrm{mg} / \mathrm{ml}$ inosine solution (Sigma) dissolved in deionized water and filter sterilized $\left(0.2-\mu \mathrm{m}\right.$ pore size)(43). The final $\mathrm{pH}$ of the liquid medium was 7.56 at $25^{\circ} \mathrm{C}$.

Escherichia coli were grown on LB-agar containing the antibiotics at standard concentrations as recommended by the primary source (Table 2) [18-22].

\subsection{Susceptibility to normal human serum}

Adult volunteers were solicited by a nurse clinician coordinator via a poster in the student union of the University of Missouri and the lobby of the Seattle Biomedical Research Institute. The goal of the study was explained and written consent to obtain $20 \mathrm{ml}$ of blood obtained. The consent form facilitated the volunteer receiving \$25 reimbursement for their time. Each subject denied taking antibiotics was without respiratory symptoms and had a blood pressure within the normal range according to the American Heart Association guidelines. Blood was obtained from 7 to 10 healthy adult volunteers at each institution. The protocol was approved by the University of Missouri-Columbia Institutional Review Board and the Institutional Review Board of the Seattle Biomedical Research Institute. Blood was allowed to clot at room temperature, and human serum was isolated aseptically, pooled, and stored at $-80^{\circ} \mathrm{C}$. 
The microtiter plate serum bactericidal assay has been described previously [23]. Briefly, bacteria were grown to $\log$ phase in CDM and 2,000 CFU were incubated for $30 \mathrm{~min}$ at $37^{\circ} \mathrm{C}$ with pooled normal human serum serially diluted in $10 \mathrm{mM}$ phosphate-buffered saline containing $4 \mathrm{mM} \mathrm{KCl}$ and $0.1 \%$ gelatin; aliquots were diluted and plated on chocolate agar to determine bacterial density. The concentration of serum that killed $50 \%$ of the inoculum was calculated after fitting the data to a Boltzmann sigmoidal curve, using the program XLfit 4.1 (ID Business Solutions, Guildford, United Kingdom), and is referred to as the 50\% inhibitory concentration $\left(\mathrm{IC}_{50 \%}\right)$ of the serum for that strain; the differences were analyzed by ANOVA calculated at the Vassar web site (VassarStats, http://vassarstats.net)

\subsection{Adherence and invasion of Human Middle Ear Epithelial Cells}

Immortalized Human middle ear epithelial cells were cultured in $250 \mu \mathrm{L}$ antibiotic-free BEGM on collagen coated 24 well microtiter plates at a density of $10^{4}$ cells per well as described previously [24]. Bacteria were sub-cultured from frozen stocks onto chocolate agar plates and incubated at $37^{\circ} \mathrm{C}$ overnight before harvesting in phosphate buffered saline containing $1 \%$ gelatin (PBS-G). Serial 10 -fold dilutions in PBS-G of each original bacterial suspension were plated on chocolate agar and the CFU enumerated after $18 \mathrm{~h}$ growth at $37^{\circ} \mathrm{C}$ to determine the viable inoculum in each assay. After the desired invasion duration aliquots of the supernatant were removed for serial dilution and plating on chocolate agar to enumerate bacteria density. Each monolayer was rinsed three times with $1 \mathrm{X}$ D-PBS (Dulbecco's PBS) and either harvested as below (for cell-associated numbers), or fresh 300 $\mu \mathrm{l}$ of medium containing $300 \mu \mathrm{g}$ gentamicin was added to each monolayer. Following 1 hour incubation at $37^{\circ} \mathrm{C}$ in medium containing gentamicin, the supernatant was aspirated and discarded and each monolayer was rinsed three times with $1.5 \mathrm{ml}$ of 1X D-PBS without calcium or magnesium. Each well was harvested by adding $1 \mathrm{ml}$ sterile 1X D-PBS containing $1 \%(\mathrm{v} / \mathrm{v})$ saponin and incubating at $37^{\circ} \mathrm{C}$ for $10 \mathrm{~min}$, followed by scrubbing with a sterile pipette tip. The cells from each well were placed in separate $1.5 \mathrm{ml}$ Eppendorf tubes and vortexed vigorously for $1 \mathrm{~min}$, after which serial 10-fold dilutions in PBS-G were performed. Aliquots $(100 \mu \mathrm{L})$ of each appropriate dilution were plated in triplicate on chocolate agar and $\mathrm{CFU}$ enumerated after $18 \mathrm{~h}$ growth at $37^{\circ} \mathrm{C}$ in atmospheric air to determine viable bacterial density. The means of three replicates, performed in triplicate, were calculated and reported.

\subsection{Adherence and Invasion of Normal Human Bronchial Epithelia Cells}

Normal human bronchial epithelial cells (NHBE, Lonza, Walkersville, MD) and cultured as a monolayer, In contrast to immortalized cell lines, NHBE cells have a phenotype closer to the epithelium in situ [25]. NHBE were seeded at a density of $5-7 \times 10^{5}$ on BD Biocoat membranes with a $4.2 \mathrm{~cm}^{2}$ growth area and a $3 \mu \mathrm{m}$ pore size in bronchial epithelial basal medium (Lonza). Bacterial inoculae were prepared in PBS-G and added to confluent NHBE monolayers at an MOI of $\sim 3: 1$ Cell supernatants and eukaryotic cells were harvested separately after 4 hours incubation, and are processed as described for the HMEEC cells. Three 'compartments' are assessed by this method: the apical supernatant indicating the amount of growth; the cell surface (associated, an index of adherence), and those invaded (i.e. intracellular, defined as being resistant to gentamicin) with each duplicate repeated twice and averages calculated and reported. 


\subsection{Adherence to HC292 cells}

To test whether OlpA2 function in adherence to eukaryotic cell, we constructed an olpA2 mutant (R3877) by the insertion of the TSTE cassette. For olpA2 mutant adherence experiments we used NCI-HC292 cells [26], a human pulmonary adenocarcinoma cell line. The experiment was conducted as described for NHBE cell line, repeated twice with the mean data reported; the significance of differences calculated with ANOVA at the Vassar web site (http://vassarstats.net).

\subsection{Colonization invasion and transcytosis of Polar NHBE Cells}

Primary normal human bronchial epithelial cells were grown to confluence in T75 flasks and then 5-7 $\times 10^{5}$ cells inoculated to the surface of the ALI on 6-well Corning Costar Transwells-COL $0.4 \mu \mathrm{m}$ membranes with a $4.2 \mathrm{~cm}^{2}$ surface area and treated as described previously [27]. We found that confluent primary human bronchial epithelial cells grown at the ALI develop a transepithelial electrical resistance (TER) of 1200 to $2000 \mathrm{~cm}^{2}$ measured with a Millicell (Millipore). We monitored the TER daily with A EVOM2 (World Precision Instruments, Sarasota FL) and when stabilized at $\sim 800 \Omega \bullet \mathrm{cm}^{2}$, serial 10 -fold dilutions R2866 and R2866modA10::kan in PBS-G were performed to yield $\sim 10^{3} \mathrm{CFU}$ in $200 \mu \mathrm{L}$ That volume was inoculated to the apical side of the membrane with diluted aliquots plated on chocolate agar and the number of colonies was counted after $18 \mathrm{~h}$ growth at $37^{\circ} \mathrm{C}$ to determine the number of viable bacteria in the inoculums. At the desired time points each insert was rinsed three times with $0.5 \mathrm{ml}$ of $1 \mathrm{X}$ D-PBS without calcium and magnesium (Dulbecco's PBS) and then incubated for $10 \mathrm{~min}$ at $37^{\circ} \mathrm{C}$ with $0.5 \mathrm{ml}$ of $1 \%$ saponin in DPBS and each insert scrapped with a plastics spatula, the lysed cells when then vigorously vortexed and the bacteria counted after plating on chocolate agar and overnight incubation at $37^{\circ} \mathrm{C}$. Bacterial density was measured in the apical compartment, those invaded (gentamicin resistant) and those transcytosed to the basal compartment. Samples were taken 12, 24, 168 and 336 hours after initial inoculation. Neither R2866 nor the modA10 mutant measurably affected the TER throughout the 14 days of incubation.

\subsection{Infant rat infection model}

Pathogen-free rats used in this study were the outbred Sprague-Dawley strain CO/BS, purchased from Charles River Laboratories (Wilmington, MA) and housed in an AALAC approved vivarium supervised by a Veterinarian. This model assess the ability of fixed macrophages to clear $H$. influenzae in the presence of specific antibody and complement. Timed-pregnant females were received at 14 days of gestation and housed individually in a pathogen free environment at $21 \pm 2^{\circ} \mathrm{C}$ and $50 \%$ relative humidity on a 7 A.M. to 7 P.M. lighting schedule. Food and water were available ad libitum. After delivery at day 21 of gestation, infant rats were pooled, randomized, and returned to the adult females in litters of 10. NTHi strains R3754 (R2866-strep ${ }^{\mathrm{R}}$ ) and the R3763 (R2866 modA10::kan knockout) were grown in room air at $37^{\circ} \mathrm{C}$ in Heme and $\beta$-NAD supplemented Brain Heart Infusion broth (sBHI) to a density of $10^{8} \mathrm{CFU} / \mathrm{ml}$, harvested by centrifugation at $5,000 \times \mathrm{g}$ at $4{ }^{\circ} \mathrm{C}$ for 15 min, resuspended in PBS-G and diluted in the same media to the desired density and stored on ice prior to inoculation: serial dilutions of the inoculum were plated on chocolate agar containing 5 units $/ \mathrm{ml}$ of bacitracin to confirm the inoculum. This allows accurate 
determination of CFU inoculated and recovered from the animals as strict aseptic conditions cannot be readily obtained with animals housed in a vivarium. For certain experiments streptomycin at $250 \mu \mathrm{g} / \mathrm{ml}$ was also added to the agar.

Bacteria were inoculated intraperitoneally in a volume of $0.1 \mathrm{ml}$ per 5-day-old infant rat as described previously [28]. Each litter with 10 randomized pups received one NTHi strain at a single inoculum and each litter housed separately with a surrogate dam. After inoculation of infant rats, the rat pups were returned to their surrogate mothers and examined at 12 hour intervals. Pups rejected by the mothers were cold and cyanotic: these animals were euthanatized by decapitation and counted as a death. Most often a pup was counted as a death when only a head was evident in the cage; rat dams ingest everything except the head when a pup is ill. Forty-eight hours after inoculation, the remaining pups all of which appeared well and were nursing, were anesthetized by inhalation of $2 \%$ di-isofluorane and $0.1 \mathrm{ml}$ of blood for culture was obtained by external jugular vein puncture. The pup was then placed in the $2 \%$ di-isofluorane until movement could not be elicited. The dams were also euthanized by with $2 \%$ di-isofluoranne. The blood was added to $0.9 \mathrm{ml}$ of sterile PBS-G and serial dilutions were plated in duplicate on chocolate agar and sBHI agar and incubated at $37^{\circ} \mathrm{C}$ in air overnight. The number of colonies were counted to determine the level of bacteremia. Prior to disposal all euthanized animals had a bilateral thoracotomy. This protocol was reviewed and approved by the Institutional Animal Care and Use Committees of the University of Missouri-Columbia and the Seattle Biomedical Research Institute.

\subsection{Transcriptomic analysis}

A 50-ml culture of R2866 or R2866modA10::kan was grown in CDM at $37^{\circ} \mathrm{C}$ in air with shaking (200rpm) to an early-log phase $\left(\mathrm{OD}_{600}\right.$ of $\left.\sim 0.3-0.4\right)$; total RNA was harvested as described previously with modification [29]. Briefly, a $25 \mathrm{ml}$ sample was mixed with $2 \mathrm{ml}$ of RNAProtect (Qiagen) to stabilize the RNA. To this, $0.2 \mathrm{ml}$ of $95 \%$ ethanol containing $5 \%$ saturated phenol $\left(4^{\circ} \mathrm{C}\right)$ was added and the culture centrifuged at $8200 \times \mathrm{g}$ at $4^{\circ} \mathrm{C}$. The pellet was resuspended in $0.8 \mathrm{ml}$ of Lysis buffer containing $1 \%(\mathrm{w} / \mathrm{v})$ SDS. The lysis mixture was incubated at $70^{\circ} \mathrm{C}$ for 6 minutes inverting the tube every 40 secs. Samples were chilled on ice and centrifuged at $21,000 \times \mathrm{g}$ for $5 \mathrm{~min}$ at $4^{\circ} \mathrm{C}$. Nucleic acid was precipitated from the supernatant by adding $1 / 10$ volume of $3 \mathrm{M}$ sodium acetate containing $1 \mathrm{mM}$ EDTA and placed at $-80^{\circ} \mathrm{C}$ for $18-24$ hours. The pellet was collected by centrifuging at $10,000 \times \mathrm{g}$ for 2 min and dissolved in RNASE-free water and the concentration estimated with a Nanodrop. The sample was then treated with RNASE-free DNASE as described in the RNA-Mini kit (Qiagen). The purity of the preparation was determined with the Agilent 2100 using the RNA 6000Lab chip; samples with a RIN value less than 4.5 were discarded. Ribosomal RNA was removed from the processed RNA using the RiboZero kit (Epicentre).

cDNA was prepared as previously described [30]. Briefly, a $20 \mu \mathrm{L}$ reaction mixture containing $7 \mu \mathrm{L}$ of template RNA, $5.5 \mathrm{mM} \mathrm{MgCl}_{2}, 500 \mu \mathrm{M}$ each deoxynucleoside triphosphate (dATP, dCTP, dGTP, and dTTP), 1 X RT buffer, $80 \mathrm{mU}$ of RNase inhibitor, a $2.5 \mu \mathrm{M}$ concentration of random hexamers, and $25 \mathrm{U}$ of MultiScribe reverse transcriptase (Applied Biosystems). The cDNA synthesis reaction mixture was incubated at $25^{\circ} \mathrm{C}$ for 10 min, followed by incubation at $48^{\circ} \mathrm{C}$ for $30 \mathrm{~min}$. The reaction was terminated by heating at 
$95^{\circ} \mathrm{C}$ for $2 \mathrm{~min}$. The cDNA $\mathrm{w}$ as sent to Nimblegen for microarray analysis, where 450,000

Haemophilus probes representing all genes of $H$. influenzae isolates, R2866, R2846, and 86-028NP were constructed with each probe consisting of a 60-mer, with 13 probes per gene; and overlapping probes in the intergenic regions. The arrays for R2866wt and the modA10::kan knockout were run in triplicate and the average signal calculated. The intrachip probe replicates were averaged prior to the analysis of the biological experimental replicates. Data were and compared between the two strains by performing individual $t$ tests using open source TMEV software [31]. Data was normalized using quantile normalization [32]. Gene expression data of strains R2866wt and the modA10::kan knockout were compared after $\log ^{2}$ transformation individual $t$ test with TMEV software (www.tm4.org) [31].. Genes whose expression was 1.5-fold different and $P \unlhd 0.05$ in the modA10::kan knockout in comparison to R2866wt were considered significant. The array data, is deposited with the Gene Expression Omnibus as GSE73349 (http://www.ncbi.nlm.nih.gov/ geo).

\subsection{Quantitative real-time PCR}

Q-PCR was performed as previously described [30]. Gene specific primers were designed using Integrated DNA Technologies Primer Quest and synthesized by Integrated DNA Technologies (Coralville, IA) and are listed in Supplementary Table 1. A $10 \mu \mathrm{L}$ reaction contained $5 \mu \mathrm{L}$ of SYBR Green Master Mix, $250 \mathrm{nM}$ of each primer and $2.5 \mu \mathrm{L}$ of cDNA. Quantification reactions were performed in quadruplicate and normalized to concurrently analyzed small subunit of $g y r A$ mRNA values in the same sample. Relative quantitation of gene expression was estimated by the $2^{-\Delta \Delta C t t}$ method [33] using wild-type R2866 cDNA as the calibrator, where $\Delta \Delta \mathrm{C}_{t}=(\mathrm{Ct}$, gene $-\mathrm{Ct}, 16 \mathrm{~s})$ time $-(\mathrm{Ct}, \mathrm{gene}-\mathrm{Ct}, 16 \mathrm{~s})$ control.

\subsection{Statistics}

The $t$ test for equal variances, and the $\mathrm{F}$ analysis of variance when they are unequal, and the $t$ test was performed using SPSS. Groups were compared using the Mann-Whit test or the Fisher exact probability test calculated online using the VassarStats Web site http:// faculty.vassar.edu/lowry/VassarStats.html.

\section{Results}

\subsection{Resistance to NHS}

Previously we developed a serum sensitivity assay in which the $\mathrm{IC}_{50 \%}$ assigns a quantitative value to the serum resistance [23]. The reference strains for this assay are Rd KW20, strain Eagan, an encapsulated type $b$ which is able to replicate in normal human serum (NHS), and strain Eagan lacking capsule that sensitizes it to NHS (Table 2). The inactivation of modA10 in the modA10::kan knockout strain has no effect on the resistance to NHS. Lipooligosaccharide (LOS) provides protection to NHS. A strain lacking functional OpsX protein (R3743) a heptosyaltransferase involved in the first steps of LOS synthesis (44) (strain R2866 ops X::TSTE), and consequently unable to produce surface LOS [35] is extremely susceptible to NHS (Table 2). The deletion of modA10 had no effect on the increased susceptibility of a double modA10/opsX mutant (R3863), R2866modA10/opsX (data not shown). Loss of the $\lg t C$ gene, encoding a galactosyltransferase, has already been 
shown to increase the sensitivity of strain R2866 to NHS [36].. Inactivation of modA10 in an lgtC negative background did not change the sensitivity to NHS (table 2).

\subsection{Strain R2866modA10::kan has increased adherence and invasion of HMEEC}

Shuto et al showed that NTHi strain 12, or a partially purified OMP P6 from that organism, activated expression of NFKB in immortalized human middle ear epithelial cells (HMEEC) [37]. In their studies, removal of LOS from NTHi strain 12 cell lysates with polymyxin did not mitigate the proinflammatory effect of the preparation. Using this system we found that the R2866 modA10::kan strain showed both greater adherence and invasion of HMEEC cells (Table 3 ) when compared to R2866wt ( $P=<0.05$ for cell association and invasion; Table 3).

\subsection{R2866modA10::kan has increased adherence and invasion of NHBE cells}

Using normal human bronchial epithelial cell monolayers, the modA10::kan mutant is significantly more adherent (cell associated) than strain R2866wt (Table 4). Although the index of invasion (invaded/cell associated) is higher for R2866wt compared to the modA10::kan mutant, the absolute numbers of invaded cells are approximately 100 -fold greater for the mutant compared to R2866wt, reflecting the increased ability to adhere by the modA10::kan mutant. Under these conditions, the number of CFU in the apical supernatant is very similar for both strains, showing the growth rate is not affected (Table 4).

\subsection{R2866modA10::kan has increased invasion and transcytosis of polar NHBE}

Primary human respiratory epithelial cells grown at air-liquid interface have a gene expression profile which closely mimics that of respiratory epithelium in vivo [38]. Certain respiratory cell lines, such as $16 \mathrm{HBEo}^{-}$form tight junctions on transwells with a low transepithelial resistance (TER) at a value four times background, $\sim 250 \Omega \cdot \mathrm{cm}^{2}(45)$. However, we could not detect a TER above background with confluent HMEEC cells grown under a variety of ALI conditions [25]. Using NHBE cells that formed a polar monolayer, indicating the presence of tight-junctions, the modA10::kan mutant strain has a marked ability to invade human bronchial epithelial cells and to transcytose the epithelial layer early in infection when compared to R2866wt 24 hours after inoculation, a feature which is maintained up to 336 hours (14 days) after initial inoculation; $P=<0.05$. (Table 5). With NHBE grown on a permeable filter the average TER in each well as $2810 \pm 50 \Omega \bullet \mathrm{cm}^{2} 24$ hours after seeding and immediately before bacterial inoculation. Throughout the experiment we could not detect a change in the TER with every other day measurements.

\subsection{R2866modA10::kan results in increased lethality in an infant rat model}

The infant rat is susceptible to invasive infection with $H$. influenzae isolated from blood or other normally sterile sites [40]. Most invasive clinical isolates are capsular type b, but strain R2866 is unique in that it lacks capsular polysaccharide, and was isolated from the blood of an immunocompetent child who was immunized with $H$. influenzae b vaccine [16] We have previously shown that the infant rat is susceptible to infection with R2866 [41].

Using this model, the R2866wt has a higher bacteremia incidence than the modA10::kan mutant (70\% total versus $35 \%)$. However, the fatality rate of rats infected with the 
modA10::kan mutant is significantly greater than that of R2866-strep ${ }^{\mathrm{r}}$ (R3754) (Table 6), with all deaths determined by the absence of a pup at 48 hours. Since the average bacterial density of the mutant in blood was not significantly different from that found with R3754 it appears that these animals developed an illness which was perceived by the dam and led to cannibalism. Thus death can result from factors other than bacteremia.

\subsection{ModA10 influenced gene expression}

We have previously demonstrated that ModA10 phase variation inR2866 did not influences expression of a number of genes that encode outer membrane proteins. [8]. To expand these studies to explore whole cell expression differences mediated by ModA10 phase variation, we performed microarray analysis on RNA preps from R2866wt and the modA10::kan knockout mutant. Genes whose expression differed by at least 1.5 -fold between the modA10::kan and R2866wt strains were considered significant. Four genes showed increased expression in the modA10::kan knockout compared with R2866 (Table 7), with seven genes showing decreased expression. Of the genes with increased transcription in the modA10 mutant two, R2866_RS04685 (ompA) and R2866_RS06645 (yeaL) are both highly conserved membrane proteins. Gene R2866_RS04675 which has a helix-turn-helix structure, is annotated as hipB (high persister B) a member of the genes in the toxinantitoxin family.

Genes with decreased expression in the $\operatorname{modA10}:$ kan knockout include those involved in haem export ( $c c m B$ and $c c m C$; R2866_RS06560 and R2866_RS06555 respectively), anaerobic respiration (Nitrite reductase; nrfA; R2866_RS06675) and metabolite biosynthesis (asnA and moaA; R2866_RS00110 and R2866_RS03925 respectively). Genes R2866_RS02455 (yjjB) and R2866_RS04270 (ycaO) encode proteins of unknown function. Both mod and res genes are significantly down-regulated in the modA10::kan strain, as would be expected due to the insertional inactivation of $\bmod$ with a kanamycin resistance cassette. The changes in expression seen on microarray analysis were confirmed by RTqPCR analysis with the primers shown in Table S1. All data is presented in Table 7

\subsection{Increased expression of olpA2_results in increased adherence}

One transcript significantly increased in R3763 is annotated as an outer membrane adhesin, olpA2 (R2866_RS04680). To investigate the role of OlpA2 in adherence, we insertionally inactivated olpA2 in strain R2866wt with a ribostamycin resistance cassette to derive strain R2866 olpA2::TSTE, and compared adherence and invasion of this olpA2::TSTE mutant to R2866wt. The experiment was conducted using HC292 cells, a human pulmonary adenocarcinoma cell line [26] and repeated twice. These cells were used as the goal was to detect adhere by the olpA2 mutant. After 4 hours incubation at $37^{\circ} \mathrm{C}$, the adherence of $\mathrm{R} 2866 w t$ in this cell line is significantly greater than that seen with the oplA2::TSTE mutant ( $\mathrm{P}<0.001)$. This indicates that expression of OlpA2 enhances NTHi adherence to eukaryotic cells, and that the increased expression of this adhesin in the $\operatorname{modA10}:$ kan mutant could lead to an enhancement of eukaryotic cell binding of a strain where modA10 is phase-varied OFF (Table 8). The olpA2::TSTE mutant shows a higher percentage of bacteria invaded the cells following this 4 hour incubation ( $\mathrm{P}<0.001$ ) when compared to R2866wt. 


\section{Discussion}

\section{1 overall virulence of $\bmod A 10$ mutant}

The data presented here indicates that phase-variation of the modA10 gene OFF (i.e., not expressed; analogous to the modA10::kan mutant characterized here) may be selected for in vitro, as loss of modA10 function increases a number of traits in strain R2866 that contribute to increased adhesion, and subsequent invasion. Wild-type R2866 with modA10 ON has increased expression of OMP 5 and OMP 6 in comparison to a derivative in which modA10 expression is mostly OFF ( 10\% ON) [8]. Both OMP P5 and OMP P6 target carcinoembryonic epitopes, but OMP 5 has specificity for macrophages (46), while OMP 6 anchors the bacterium to CECAM epitopes in mucin (47). Strains which cause infections limited to mucosal surfaces are resistant to low levels of serum (48). Invasive NTHi strains possess mechanisms to block the action of complement protein $\mathrm{C} 3$ which inhibits the lytic reaction (49); with the severity of invasive NTHi disease correlating with higher levels of serum resistance (50). The high level of serum resistance seen with strain R2866 permits it to cause invasive infections., This resistance is not increased in the modA10::kan mutant, implying that resistance to NHS is an innate ability of this strain, due to the expression of $\operatorname{lgt} C$ which is not affected by modA10 phase variation. We observed increased adhesive, invasive, and transcytosis capabilities with R2866modA10::kan compared to R2866wt, using both human airway and human middle ear epithelial cell lines, We postulate that in human patients the modA10 OFF state may be selected providing an accessory mechanism to cause disease.

\subsection{Adherence and Transcytosis}

Bacteria were also found intracellularly at higher CFU with the modA10::kan mutant using our human cell lines, indicating intracellular survival may be facilitated by the modA10 phasevarion OFF. Previous studies have shown that NTHi can survive within the lysosomal compartment in eukaryotic cells [51], that these NTHi cells were viable, and that intercellular residence shields the bacteria from the host immune system and antibiotics, sustaining mucosal colonization when the bacteria emerge apically [52]. Transcytosis into the submucosa is a mechanism that can shield the bacterium from mucosal host defense, and lead to bacterial replication in blood.. NTHi strain 12 has been shown to bind to a (2-3) sialic acid on the surface of $M$ cells in the guinea pig conjunctiva and then invaded [47]. Some or all of these traits appear to be enhanced by $\operatorname{modA10}$ phase-varying OFF, as the modA10::kan mutant shows significantly increased ability to adhere, invade cells, and pass through polar epithelial cells.

Strain R2866 also contains the adhesin Hia [54], which is present in approximately $25 \%$ of all NTHi isolates. Hia expression is subject to phase variable expression by variation in length of a poly-thymidine tract located in the promoter region of the hia gene [5] and although Hia expression is not influenced by modA10 phase variation, the importance of Hia in initial colonization of the chinchilla nasopharynx [51] could lead to a synergism with the adhesins regulated by modA10 phase variation. 


\section{3 modA10 modulated gene expression}

There are 1244 targets, 5'-CCTA ${ }^{\mathrm{m}} \mathrm{C}-3$, in the $\mathrm{R} 2866$ genome with only 384 motifs in coding regions [8]. The only phenotypic difference seen in cultures with predominantly OFF $(\sim 90 \%)$ in comparison to R2866wt (90\% ON) were increased expression of OMP-P5 and OMP-P6 when modA10 was ON [8].

\subsection{Transcriptome of R2866wt compared to R2866modA10::kan}

4.4.1 Genes with increased transcription in R2866modA10::kan-Four genes whose transcription was increased in the modA10::kan mutant are predicted to encode bacterial surface proteins. One of the gene products, OlpA2 was confirmed to mediate adherence and invasion of a human adenocarcinoma cell line (Table 8). This protein is currently uncharacterized, and is widely conserved in NTHi strains. Evidence presented here shows it has an important role in adhesion and invasion of eukaryotic cells. The high level of conservation amongst NTHi lends support to investigation of this protein as a possible vaccine candidate. OlpA2 is distinct from the OlpA1 protein encoded by R2866_RS06150 (YP_005827896.1), sharing only 34\% amino acid identity. However, both proteins are members of the opacity porin family. Porins have been shown to be important for antimicrobial resistance in the pathogenic Neisseria [48] and Escherichia coli [49,50]. The putative amino acid sequence of OlpA2 has no significant homology to the sequence of OapA in Rd KW20 [51]. OlpA2 has homology to a Moraxella catarrhalis outer membrane protein (encoded by olpA) which is involved in adherence [52] and resistance to normal human serum [53].

4.4.2 Genes with decreased transcription in R2866modA10::kan-Of the genes whose transcription is decreased in the modA10::kan mutant the $\mathrm{ccmB}$ and $\mathrm{ccmC}$ are part of the heme export pathway. Nitrite reductase ( $r r f A)$ is a cytochrome dependent (C552) periplasmic nitrite reductase producing ammonia and generating energy under anaerobic conditions. AsnA is annotated as a transcriptional regulator of asparagine biosynthesis; its decreased expression correlates with the decreased expression of $n f r A$. MoaA is predicted to function as a molybdenum cofactor biosynthetic protein, which contains several iron-sulphur clusters as cofactors. A concerted ability to conserve heme and/or iron by decreasing the expression of proteins that either export them or require them as cofactors could give the modA10::kan mutant an advantage in host niches where iron is restricted.

Considering all the phenotypic properties of the modA10 mutant of NTHi strain R2866 it appears that a switch from the ON reading frame to OFF (or inactivated with an antibiotic cassette) results in the expression of genes permitting continuation of the bacterium's lifestyle: adherence to respiratory epithelial cells followed by replication at that site, cellular invasion, and transcytosis of the epithelium. These abilities appear to be enhanced by the phase-varying of modA10 OFF, and are likely in part due to increased expression of proteins involved in cellular adhesion, in which OlpA2 appears to play a key role. Replication in the sub-epithelial compartment, likely enhanced by an ability to conserve iron, would permit increased epithelial colonization through exocytosis, and systemic infection by evading host defenses. 


\section{Conclusions}

H.influenzae R2866 is nontypeable but caused meningitis in an 18 month old child who was documented to be immunocompetent. It replicated in the blood through the expression of $\operatorname{lgt} C$ a gene which encodes surface localized Gala1-4ßGal an epitope which mimics the human $\mathrm{pk}^{\mathrm{c}}$ blood group, resulting in the strain not recognized as foreign. Adherence to eukaryotic cells is necessary for invasion, but certain adhesins, eg HMW1/2A, Protein D are expressed at low levels in this strain [8]. We found that loss of function of a DNA methylase, M.Hin2866I (R2866modA10::kan) increases the adherence, invasion and transcytosis of human epithelial cells. Virulence in the infant rat model was increased but in an anomalous manner.

\section{Supplementary Material}

Refer to Web version on PubMed Central for supplementary material.

\section{Acknowledgments}

The authors are grateful for the discussions with Yogitha Srikhanta regarding modA10 phase variation in Haemophilus influenzae strain R2866. This work was supported by NIH grant AI 46512.

\section{References}

1. Ewrin AL, Smith AL. Nontypeable Haemophilus influenzae: understanding virulence and commensal behavior. Trends in Microbiology. 2007; 15(8):355-362. [PubMed: 17600718]

2. Poole J, Foster E, Chaloner K, Hunt J, Jennings MP, Bair T, et al. Analysis of nontypeable Haemophilus influenzae phase variable genes during experimental human nasopharyngeal colonization. J Infect Dis. 2013; 208:720-7. [PubMed: 23715658]

3. Ren Z, Jin H, Whitby PW, Morton DJ, Stull TL. Role of CCAA nucleotide repeats in regulation of hemoglobin and hemoglobin-haptoglobin binding protein genes of Haemophilus influenzae. J Bacteriol. 1999; 181(18):5865-70. [PubMed: 10482534]

4. Richardson AR, Stojiljkovic I. HmbR, a hemoglobin-binding outer membrane protein of Neisseria meningitidis, undergoes phase variation. J Bacteriol. 1999; 181(7):2067-74. [PubMed: 10094683]

5. Atack JM, Winter LE, Jurcisek JA, Bakaletz LO, Barenkamp SJ, Jennings MP. Selection and counter-selection of Hia expression reveals a key role for phase-variable expression of this adhesin in infection caused by non-typeable Haemophilus influenzae. J Infect Dis. 2015 10.1093/infdis/ jiv103.

6. Moxon ER, Thaler DS. Microbial genetics. The tinkerer's evolving tool-box. Nature. 1997; 387(6634):659, 61-2. [PubMed: 9192885]

7. Srikhanta YN, Fox KL, Jennings MP. The phasevarion: phase variation of type III DNA methyltransferases controls coordinated switching in multiple genes. Nat Rev Micro. 2010; 8(3): 196-206.

8. Atack JM, Srikhanta YN, Fox KL, Jurcisek JA, Brockman KL, Clark TA, et al. A biphasic epigenetic switch controls immunoevasion, virulence and niche adaptation in non typeable Haemophilus influenzae. Nat Commun. 2015; 6:7828. 10.11038/ncomms8828. [PubMed: 26215614]

9. Srikhanta YN, Maguire TL, Stacey KJ, Grimmond SM, Jennings MP. The phasevarion: A genetic system controlling coordinated, random switching of expression of multiple genes. Proc Natl Acad Sci U S A. 2005; 102(15):5547-51. [PubMed: 15802471]

10. Seib KL, Jen FE, Tan A, Scott AL, Kumar R, Power PM, et al. Specificity of the ModA11, ModA12 and ModD1 epigenetic regulator N6-adenine DNA methyltransferases of Neisseria meningitidis. Nucleic Acids Res. 2015; pii:gkv219. 
11. Seib KL, Pigozzi E, Muzzi A, Gawthorne JA, Delany I, Jennings MP, et al. A novel epigenetic regulator associated with the hypervirulent Neisseria meningitidis clonal complex 41/44. FASEB J. 2011; 25(10):3622-33. [PubMed: 21680891]

12. Srikhanta YN, Dowideit SJ, Edwards JL, Falsetta ML, Wu H-J, Harrison OB, et al. Phasevarions mediate random switching of gene expression in pathogenic Neisseria. PLoS Pathog. 2009; 5(4):e1000400. [PubMed: 19390608]

13. Srikhanta YN, Gorrell RJ, Steen JA, Gawthorne JA, Kwok T, Grimmond SM, et al. Phasevarion mediated epigenetic gene regulation in Helicobacter pylori. PLoS One. 2011; 6(12):e27569. [PubMed: 22162751]

14. Blakeway LV, Power PM, Jen FE, Worboys SR, Boitano M, Clark TA, et al. ModM DNA methyltransferase methylome analysis reveals a potential role for Moraxella catarrhalis phasevarions in otitis media. FASEB J. 2014; 28(12):5197-207. [PubMed: 25183669]

15. Gawthorne JA, Beatson SA, Srikhanta YN, Fox KL, Jennings MP. Origin of the diversity in DNA recognition domains in phasevarion associated modA genes of pathogenic Neisseria and Haemophilus influenzae. PLoS One. 2012; 7(3):e32337. [PubMed: 22457715]

16. Nizet V, Colina KF, Almquist JR, Rubens CE, Smith AL. A virulent nonencapsulated Haemophilus influenzae. J Iinfect Dis. 1996; 173(1):180-6.

17. Coleman HN, Daines DA, Jarisch J, Smith AL. Chemically defined media for growth of Haemophilus influenzae strains. J Clin Microbiol. 2003; 41(9):4408-10. [PubMed: 12958278]

18. Whitby PW, Morton DJ, Stull TL. Construction of antibiotic resistance cassettes with multiple paired restriction sites for insertional mutagenesis of Haemophilus influenzae. FEMS Microbiol Lett. 1998; 158(1):57-60. [PubMed: 9453156]

19. Chang AC, Cohen SN. Construction and characterization of amplifiable multicopy DNA cloning vehicles derived from the P15A cryptic miniplasmid. J Bacteriol. 1978; 134(3):1141-56. [PubMed: 149110]

20. Sharetzsky C, Edlind TD, LiPuma JJ, Stull TL. A novel approach to insertional mutagenesis of Haemophilus influenzae. J Bacteriol. 1991; 173(4):1561-4. [PubMed: 1847369]

21. Martinez E, Bartolome B, de la Cruz F. pACYC184-derived cloning vectors containing the multiple cloning site and lacZ alpha reporter gene of pUC8/9 and pUC18/19 plasmids. Gene. 1988; 68(1):159-62. [PubMed: 2851489]

22. Daines DA, Smith AL. Design and construction of a Haemophilus influenzae conjugal expression system. Gene. 2001; 281(1-2):95-102. [PubMed: 11750131]

23. Erwin AL, Nelson KL, Mhlanga-Mutangadura T, Bonthuis PJ, Geelhood JL, Morlin G, et al. Characterization of genetic and phenotypic diversity of invasive nontypeable Haemophilus influenzae. Infect Immun. 2005; 73(9):5853-63. [PubMed: 16113304]

24. Chun YM, Moon SK, Lee HY, Webster P, Brackmann DE, Rhim JS, et al. Immortalization of normal adult human middle ear epithelial cells using a retrovirus containing the E6/E7 genes of human papillomavirus type 16. Ann Otol Rhinol Laryngol. 2002; 111(6):507-17. [PubMed: 12090706]

25. Widdicombe JH, Sachs LA, Morrow JL, Finkbeiner WE. Expansion of cultures of human tracheal epithelium with maintenance of differentiated structure and function. Biotechniques. 2005; 39(2): 249-55. [PubMed: 16116798]

26. van Schilfgaarde M, van Ulsen P, Eijk P, Brand M, Stam M, Kouame J, et al. Characterization of adherence of nontypeable Haemophilus influenzae to human epithelial cells. Infect Immun. 2000; 68(8):4658-65. [PubMed: 10899870]

27. Daines DA, Cohn LA, Coleman HN, Kim KS, Smith AL. Haemophilus influenzae Rd KW20 has virulence properties. J Med Microbiol. 2003; 52(Pt 4):277-82. [PubMed: 12676864]

28. Smith AL, Smith DH, Averill DR Jr. Marino J, Moxon ER. Production of Haemophilus influenzae b meningitis in infant rats by intraperitoneal inoculation. Infect Immun. 1973; 8(2):278-90. [PubMed: 4542033]

29. Jahn CE, Charkowski AO, Willis DK. Evaluation of isolation methods and RNA integrity for bacterial RNA quantitation. J Microbiol Methods. 2008; 75(2):318-24. [PubMed: 18674572] 
30. VanWagoner TM, Whitby PW, Morton DJ, Seale TW, Stull TL. Characterization of three new competence-regulated operons in Haemophilus influenzae. J Bacteriol. 2004; 186(19):6409-21. [PubMed: 15375121]

31. Saeed AI, Sharov V, White J, Li J, Liang W, Bhagabati N, et al. TM4: a free, open- source system for microarray data management and analysis. BioTechniques. 2003; 34(2):374-8. [PubMed: 12613259]

32. Bolstad BM, Irizarry RA, Astrand M, Speed TP. A comparison of normalization methods for high density oligonucleotide array data based on variance and bias. Bioinformatics. 2003; 19(2):18593. [PubMed: 12538238]

33. Livak KJ, Schmittgen TD. Analysis of relative gene expression data using real-time quantitative PCR and the 2(-Delta Delta C(T)) Method. Methods. 2001; 25(4):402-8. [PubMed: 11846609]

34. Gronow S, Brabetz W, Lindner B, Brade H. OpsX from Haemophilus influenzae Represents a Novel Type of Heptosyltransferase I in Lipopolysaccharide Biosynthesis. J Bacteriol. 2005; 187(17):6242-7. [PubMed: 16109967]

35. Ho DK, Ram S, Nelson KL, Bonthuis PJ, Smith AL. lgtC expression modulates resistance to C4b deposition on an invasive nontypeable Haemophilus influenzae. J Immunol. 2007; 178(2):100212. [PubMed: 17202363]

36. Erwin AL, Allen S, Ho DK, Bonthius PJ, Jarisch J, Nelson KL, et al. Role of lgtC in Resistance of Nontypeable Haemophilus influenzae Strain R2866 to Human Serum. Infect Immun. 2006; 74(11): 6226-35. [PubMed: 16966407]

37. Shuto T, Xu H, Wang B, Han J, Kai H, Gu XX, et al. Activation of NF-kappa B by nontypeable Hemophilus influenzae is mediated by toll-like receptor 2-TAK1-dependent NIK IKK alpha /beta-I kappa B alpha and MKK3/6-p38 MAP kinase signaling pathways in epithelial cells. Proc Natl Acad Sci U S A. 2001; 98(15):8774-9. [PubMed: 11438700]

38. Pezzulo AA, Starner TD, Scheetz TE, Traver GL, Tilley AE, Harvey BG, et al. The air-liquid interface and use of primary cell cultures are important to recapitulate the transcriptional profile of in vivo airway epithelia. Am J Physiol Lung Cell Mol Physiol. 2011; 300(1):L25-31. [PubMed: 20971803]

39. Forbes B, Shah A, Martin GP, Lansley AB. The human bronchial epithelial cell line 2003;257(1-2):161-7.16HBE14o-as a model system of the airways for studying drug transport. Int J Pharm. 2003; 257(1-2):161-7. [PubMed: 12711171]

40. Weller PF, Smith AL, Anderson P, Smith DH. The role of encapsulation and host age in the clearance of Haemophilus influenzae bacteremia. J Infect Dis. 1977; 135(1):34-41. [PubMed: 299866]

41. Tsao D, Nelson KL, Kim D, Smith AL. Infant rat infection modifies phenotypic properties of an invasive nontypeable Haemophilus influenzae. Microbes Infect. 2012; 14(6):509-16. [PubMed: 22222846]

42. Nakamura S, Shchepetov M, Dalia AB, Clark SE, Murphy TF, Sethi S, et al. Molecular Basis of Increased Serum Resistance among Pulmonary Isolates of Non-typeable Haemophilus influenzae. PLoS Pathog. 2011; 7(1):e1001247. [PubMed: 21253576]

43. Hallstrom T, Resman F, Ristovski M, Riesbeck K. Binding of complement regulators to invasive nontypeable Haemophilus influenzae isolates is not increased compared to nasopharyngeal isolates, but serum resistance is linked to disease severity. J Clin Microbiol. 2010; 48(3):921-7. [PubMed: 20089757]

44. Hallstrom T, Riesbeck K. Haemophilus influenzae and the complement system. Trends Microbiol. 2010; 18(6):258-65. [PubMed: 20399102]

45. Morey P, Cano V, Marti-Lliteras P, Lopez-Gomez A, Regueiro V, Saus C, et al. Evidence for a non-replicative intracellular stage of nontypable Haemophilus influenzae in epithelial cells. Microbiology. 2011; 157(Pt 1):234-50. [PubMed: 20929955]

46. Salcedo SP, Cid VJ. Nontypable Haemophilus influenzae: an intracellular phase within epithelial cells might contribute to persistence. Microbiology. 2011; 157(Pt 1):1-2. [PubMed: 21186322]

47. Petris CK, Golomb M, Phillips TE. Bacterial transcytosis across conjunctival M cells. Invest Ophthalmol Vis Sci. 2007; 48(5):2172-7. [PubMed: 17460276] 
48. Peak IR, Jennings CD, Jen FE, Jennings MP. Role of Neisseria meningitidis PorA and PorB expression in antimicrobial susceptibility. Antimicrob Agents Chemother. 2014; 58(1):614-6. [PubMed: 24145542]

49. Dé E, Baslé A, Jaquinod M, Saint N, Malléa M, Molle G, et al. A new mechanism of antibiotic resistance in Enterobacteriaceae induced by a structural modification of the major porin. Molecular Microbiology. 2001; 41(1):189-98. [PubMed: 11454211]

50. Curtis NAC, Eisenstadt RL, Turner KA, White AJ. Porin-mediated cephalosporin resistance in Escherichia coli K-12. J Antimicrob Chemotherap. 1985; 15(5):642-4.

51. Weiser JN, Chong ST, Greenberg D, Fong W. Identification and characterization of a cell enve lope protein of Haemophilus influenzae contributing to phase variation in colony opacity and nasopharyngeal colonization. Mol Microbiol. 1995; 17(3):555-64. [PubMed: 8559074]

52. Brooks MJ, Laurence CA, Hansen EJ, Gray-Owen SD. Characterization of the Moraxella catarrhalis opa-like protein, OlpA, reveals a phylogenetically conserved family of outer membrane proteins. J Bacteriol. 2007; 189(1):76-82. [PubMed: 17041038]

53. Bernhard S, Fleury C, Su YC, Zipfel PF, Koske I, Nordstrom T, et al. Outer Membrane Protein OlpA Contributes to Moraxella catarrhalis Serum Resistance via Interaction With Factor H and the Alternative Pathway. J Infect Dis. 2014; 210(8):1306-10. [PubMed: 24771863]

54. St Geme JW 3rd, Kumar VV, Cutter D, Barenkamp SJ. Prevalence and distribution of the hmw and hia genes and the HMW and Hia adhesins among genetically diverse strains of nontypeable Haemophilus influenzae. Infect Immun. 1998; 66(1):364-8. [PubMed: 9423882] 


\section{Highlights}

- Haemophilus influenzae commonly a commensal but occasionally pathogenic.

- Adherence and invasion of respiratory cells mediates virulence

- Phase variable methylase modulates virulence variation due to slipped strand mis-pairing

- Inactivation of $\operatorname{modA10}$ methylase increases expression of virulence 


\section{Table 1}

Escherichia coli strains and plasmids used in this study

\begin{tabular}{|l|l|}
\hline pGEM-Teasy & Promega \\
\hline DH5a/pEM7/Zeo & Invitrogen \\
\hline DH5a/pSpec & aad; Whitby et al, 1998 [19] \\
\hline HB101/pACYC184 & cat; Chang et al, 1978 [20] \\
\hline DH5a/TSTE & aph; ribostamycin resistance; Sharetzsky et al, 1991 [21] \\
\hline DH5a/pSU2718 & cat; Martinez et al, 1988 [22] \\
\hline DH5a/pUC 4 cat & Daines \& Smith, 2001 [23] \\
\hline DD12/pDD514 & Daines \& Smith, 2001 [23] \\
\hline
\end{tabular}




\section{Table 2}

IC50\% using Normal Human Serum

\begin{tabular}{|l|c|c|c|}
\hline & & \multicolumn{2}{|c|}{ IC50\% } \\
\hline Strain & n & mean & Range \\
\hline R2866wt & 84 & $14.45^{*}$ & $5.74-26.08$ \\
\hline R2866 modA10::kan & 4 & $16.44^{*}$ & $6.82-25.0$ \\
\hline R2866opsX::TSTE & 4 & 0.50 & $0.05-2.22$ \\
\hline R2866IgtC::cat & 4 & 1.74 & $0.82-2.88$ \\
\hline R2866IgtC:: spec; modA10::kan & 4 & 1.86 & 0.882 .24 \\
\hline Rd KW20 & 79 & 0.71 & $0.34-3.01$ \\
\hline E1a & 42 & 22.60 & $18.4->25$ \\
\hline E1a $\Delta$ cap gene cluster & 16 & 1.24 & $0.60-1.42$ \\
\hline
\end{tabular}

$\mathrm{n}=$ number of replicates

$\boldsymbol{\Delta}$ Different from E1a at $\mathrm{p}<.001$

* Not significantly different than E1a $P>0.24$ 
Table 3

challenge of HMEEC cells with strains R2866wt and R2866 modA10::kan

\begin{tabular}{|l|c|c|c|}
\hline & R2866wt & R2866modA10::kan & Rd KW20 \\
\hline Inoculum (CFU) & $3.87 \times 10^{6}$ & $7.51 \times 10^{6}$ & $3.25 \times 10^{6}$ \\
\hline Apical compartment (CFU) & $5.80 \times 10^{7}$ & $4.80 \times 10^{7}$ & $5.05 \times 10^{7}$ \\
\hline Cell associated (CFU) & $4.30 \times 10^{6}$ & $1.95 \times 10^{7}$ & $4.15 \times 10^{5}$ \\
\hline Invaded (CFU) & $9.50 \times 10^{3}$ & $9.75 \times 10^{4}$ & $1.02 \times 10^{2}$ \\
\hline Cell associated; \% of total CFU & 6.90 & $28.85^{*}$ & 0.82 \\
\hline Invaded; \% of cell associated & 0.22 & $0.50^{*}$ & 0.02 \\
\hline
\end{tabular}

Total CFU $=$ Apical compartment + cell associated + invaded CFU

Statistical significance compared to R2866wt; $\mathrm{P}=<0.05$ 


\section{Table 4}

Adherence to NHBE monolayer

\begin{tabular}{|l|c|c|c|}
\hline & Rd KW20 & R2866wt & R3763 \\
\hline Inoculum (CFU) & $3.25 \times 10^{6}$ & $3.87 \times 10^{6}$ & $5.80 \times 10^{6}$ \\
\hline Apical supernatant (CFU) & $5.05 \times 10^{7}$ & $1.23 \times 10^{7}$ & $1.98 \times 10^{7}$ \\
\hline Cell associated (CFU) & $4.15 \times 10^{5}$ & $2.49 \times 10^{3}$ & $4.79 \times 10^{5}$ \\
\hline Invaded (CFU) & $1.02 \times 10^{2}$ & $7.13 \times 10^{2}$ & $5.25 \times 10^{4}$ \\
\hline Cell associated; \% of total CFU & 0.082 & 0.02 & $2.36^{*}$ \\
\hline Invaded; \% of cell associated & 0.024 & 28.63 & 10.96 \\
\hline
\end{tabular}

Total CFU = Apical compartment + cell associated + invaded CFU

* Statistical significance compared to R2866wt; $\mathrm{P}=<0.05$ 
Table 5

Colonization, invasion and transcytosis of polar NHBE

\begin{tabular}{|l|c|c|c|c|}
\hline Average number of bacteria at the time indicated & & & & \\
\hline R2866wt & $\mathbf{1 2 h r s}$ & $\mathbf{2 4 h r s}$ & $\mathbf{1 6 8 h r s}$ & $\mathbf{3 3 6 h r s}$ \\
\hline Apical supernatant (CFU) & $1.55 \times 10^{7}$ & $2.15 \times 10^{8}$ & $9.25 \times 10^{7}$ & $2.45 \times 10^{6}$ \\
\hline Invaded (CFU) & 0 & $2.04 \times 10^{4}$ & $1.50 \times 10^{5}$ & $2.25 \times 10^{6}$ \\
\hline Transcytosed (CFU) & 0 & $1.20 \times 10^{2}$ & $8.42 \times 10^{7}$ & $1.83 \times 10^{7}$ \\
\hline R2866 modA10::kan & & & & \\
\hline Apical supernatant (CFU) & $6.82 \times 10^{8}$ & $4.40 \times 10^{8}$ & $5.20 \times 10^{8}$ & $6.82 \times 10^{7}$ \\
\hline Invaded (CFU) & 0 & $5.28 \times 10^{5^{*}}$ & $1.69 \times 10^{7^{*}}$ & $4.95 \times 10^{6}$ \\
\hline Transcytosed (CFU) & 0 & $8.75 \times 10^{6}$ & $1.08 \times 10^{8}$ & $1.73 \times 10^{7}$ \\
\hline
\end{tabular}

Initial inoculum of R2866 was $5.87 \times 10^{3} \mathrm{CFU}$, while for strain R2866modA10::kan it was $7.51 \times 10^{3}$

* Statistical significance compared to R2866wt; $P=<0.05$ 


\section{Table 6}

Virulence in infant rats after intraperitoneal inoculation

\begin{tabular}{|l|c|c|c|c|c|c|c|}
\hline Strain & inoculum & $\mathbf{n}$ & \# bacteremic & \% bacteremic & \# dead & \% death (\#dead/n) & average CFU/ml blood \\
\hline R2866-strep $^{\text {R }}$ & $2.90 \times 10^{6}$ & 10 & 7 & 70 & 2 & $20^{*}$ & $4.30 \times 10^{6}$ \\
\hline R32866modA10::kan & $1.70 \times 10^{6}$ & 20 & 7 & 35 & 13 & $65^{*}$ & $3.33 \times 10^{6}$ \\
\hline E1a & $1.72 \times 10^{6}$ & 10 & 5 & 50 & 5 & 50 & $1.81 \times 10^{7}$ \\
\hline Rd KW20 & $2.4 \times 10^{6}$ & 10 & 2 & 20 & 0 & 0 & $1.69 \times 10^{5}$ \\
\hline
\end{tabular}

The difference in the fatality rate between R2866modA10::kan and the wild type is statistically significant; $P=0.0201$ by two-tailed t test. 
Table 7

Results of R2866wt vs R2866modA10::kan gene expression differences

\begin{tabular}{|l|l|c|c|c|}
\hline \multirow{2}{*}{ Gene } & \multirow{2}{*}{ NCBI annotation } & \multicolumn{2}{|c|}{ microarray } & \multirow{2}{*}{ RTqPCR } \\
\cline { 3 - 4 } & & fold change & p value & \\
\hline Increased expression in R2866 modA10::kan \\
\hline ompA-like & R2866_RS04685 & 3.32 & 0.0067 & 5.68 \\
\hline olpA2 & R2866_RS04680 & 2.69 & 0.0021 & 6.43 \\
\hline yeaL & R2866_RS06645 & 1.5 & 0.040 & 2.34 \\
\hline hipB & R2866_RS04675 & 1.44 & 0.0095 & 1.86 \\
\hline Decreased expression in R2866 modA10::kan & & \\
\hline yjjB & R2866_RS02455 & -1.45 & 0.030 & -1.62 \\
\hline ccmB & R2866_RS06560 & -1.52 & 0.0049 & -1.74 \\
\hline nrfA & R2866_RS06675 & -1.53 & 0.036 & -2.08 \\
\hline moaA & R2866_RS03925 & -1.75 & 0.048 & -2.34 \\
\hline$y c a O$ & R2866_RS04270 & -1.65 & 0.010 & -1.46 \\
\hline ccm $C$ & R2866_RS06555 & -1.75 & 0.029 & -3.14 \\
\hline asnA & R2866_RS00110 & -3.29 & 0.011 & -4.85 \\
\hline res* & R2866_RS06725 & -121.31 & 0.0016 & -248.62 \\
\hline mod $*$ & R2866_RS06730 & -137.89 & 0.000063 & -356.66 \\
\hline
\end{tabular}


Table 8

Contribution of OlpA2 to adherence and invasion of HC292 cells

\begin{tabular}{|l|c|c|}
\hline & R2866 & R2866olpA2::TSTE \\
\hline Inoculum (CFU) & $5.13 \times 10^{6^{*}}$ & $3.10 \times 10^{6^{*}}$ \\
\hline Apical compartment & $3.23 \times 10^{7^{*}}$ & $9.40 \times 10^{6^{*}}$ \\
\hline Cell associated; \% of total (CFU) & $1.82 \times 10^{7^{*}}$ & $3.78 \times 10^{5^{*}}$ \\
\hline Invaded (CFU) & $6.14 \times 10^{4^{*}}$ & $1.27 \times 10^{3^{*}}$ \\
\hline Cell associated; \% of total CFU & $44.32 \pm 4.20$ & $3.87 \pm 2.64$ \\
\hline Invaded; \% of cell associated & $0.295 \pm 0.065$ & $2.11 \pm 0.83^{\#}$ \\
\hline
\end{tabular}

*average of two replicates

**

cell associated (adherent) number is greater at $\mathrm{p}<0.001$

${ }^{\#}$ the number of invaded bacteria is greater at $\mathrm{p}<0.001$ 